\title{
In Vitro Cardiomyogenic Potential of Human Amniotic Fluid Stem Cells
}

\author{
Xuan Guan", Dawn M. Delo*, Anthony Atala, and Shay Soker\# \\ Wake Forest Institute for Regenerative Medicine, Wake Forest University Health Sciences, \\ Medical Center Blvd, Winston-Salem, NC 27157
}

Xuan Guan: xuanguan@gmail.com; Dawn M. Delo: dawn.delo@gmail.com; Anthony Atala: aatala@wfubmc.edu; Shay

Soker: ssoker@wfubmc.edu

\section{Abstract}

Stem cell therapy for damaged cardiac tissue is currently limited by a number of factors, including the inability to obtain sufficient cell numbers, the potential tumorigenicity of certain types of stem cells, and the possible link between stem cell therapy and the development of malignant arrhythmias. In this study, we investigated whether human amniotic fluid-derived stem (hAFS) cells could be a potential source of cells for cardiac cell therapy by testing the in vitro differentiation capabilities. Undifferentiated hAFS cells express several cardiac genes, including the transcription factor mef2, the gap junction connexin 43 , and $\mathrm{H}$ - and $\mathrm{N}$-cadherin. A 24-hour incubation with 5-aza-2'-deoxycytidine (5-AZA-dC) induced hAFS cell differentiation along the cardiac lineage. Evidence for this differentiation included morphological changes, up-regulation of cardiac-specific genes (cardiac troponin I and cardiac troponin T) and redistribution of connexin43, as well as down-regulation of the stem cell marker SRY-box 2 (sox2). When cocultured with neonatal rat cardiomyocytes (NRCs), hAFS cells formed both mechanical and electrical connections with the NRCs. Dye transfer experiments showed that calcein dye could be transferred from NRCs to hAFS cells through cellular connections. The gap junction connexin 43 likely involved in the communication between the two cell types, because 12-O-

Tetradecanoylphorbol 13-acetate (TPA) could partially block cellular crosstalk. We conclude that hAFS cells can be differentiated into a cardiomyocyte-like phenotype and can establish functional communication with NRCs. Thus, hAFS cells may potentially be used for cardiac cell therapy.

\section{Keywords}

stem cells; cell differentiation; myocytes; cardiac; amniotic fluid stem cells; coculture techniques

\section{Introduction}

Heart disease, including acute myocardial infarction and congestive heart failure, remains the leading cause of morbidity in the world. New treatment modalities such as new drugs, nutrition and lifestyle change have greatly improved the mortality rate and the quality of life for cardiac patients. However, the damage to the cardiac muscle remains irreversible due, in part, to the low regenerative potential of postnatal cardiomyocytes.

\footnotetext{
\#To Whom Correspondence should be addressed.

* These authors contributed equally to this work

Disclosure: None.
} 
Cell therapy has recently been used to treat a variety of degenerative diseases, including heart disease. Different types of stem and progenitor cells have been differentiated into cardiomyocyte-like cells in vitro (Smits et al., 2009; Xu, Police, Rao, \& Carpenter, 2002; Zhao et al., 2005), suggesting that they could be used for cell therapy to replace damaged cardiac muscle tissue. In parallel, studies have shown that secretion of cytokines from injected cells resulted in improvement of cardiac function which continued well after the injected cells could no longer be detected in the tissue (see review (Laflamme \& Murry, 2005)). Despite these encouraging results, reports have shown that skeletal myoblasts injected into the myocardium may increase the incidence of post-ischemic arrhythmias (Leobon et al., 2003; Menasche et al., 2008). In addition, other studies have demonstrated that the positive effects of injected bone marrow-derived mesenchymal stem cells were only transient (Meyer et al., 2006).

To address some of the problems associated with cellular therapies for heart disease, alternative cell sources have been suggested. Embryonic stem cells and induced pluripotent stem cells (iPS), for example, can spontaneously form beating cells with a cardiac-like phenotype in vitro. These patient-specific iPS cells could be used to avoid the ethical and immunogenicity concerns of embryonic stem cells (Takahashi \& Yamanaka, 2006; Yu et al., 2007). However, the tumorigenic potential of these cells prevents their clinical use (He et al., 2009).

We have recently described a new type of stem cell that can be isolated from human amniotic fluid, termed human amniotic fluid stem cells (hAFS) cells (De Coppi et al., 2007). These cells are multipotent and are highly proliferative. In addition, undifferentiated hAFS cells express several cardiac associated genes. The present study was designed to test whether hAFS cells could be differentiated along the cardiac lineage. We show that when hAFS cells are induced with 5-aza-2'-deoxycytidine, expression of several cardiac genes are increased. Further, we have demonstrated that hAFS cells can form functional cell-cell communication channels with neonatal cardiomyocytes in an in vitro co-culture experiment. Together, these results suggest that hAFS cells may serve as a new and valuable cell source for cardiac cell therapy.

\section{Materials and Methods}

\section{1. hAFS cell culture and cardiac differentiation}

Multipotent subpopulation of c-kit+ progenitor cells were isolated from human amniotic fluid as previously described (De Coppi et al., 2007). These hAFS cells were grown on nontreated plastic dishes in culture medium consisting of minimum essential alpha-medium, embryonic stem cell certified fetal bovine serum (15\%), L-glutamine (1\%), antibiotics (1\%) (Invitrogen), and Chang Medium ${ }^{\circledR}$ B (18\%) and C (2\%) (Irvine Scientific). For cardiogenic induction, hAFS cells were plated at 3,000 cells $/ \mathrm{cm}^{2}$ on tissue culture dishes in growth medium for 12 hours for attachment, then switched to differentiation medium consists of low-glucose DMEM supplemented with $10 \%$ horse serum, $10 \%$ Fetal Bovine Serum, and $1 \%$ Penicillin/Streptomycin. In the first 24 hours, $10 \mu \mathrm{M}$ 5-aza-2'-deoxycytidine (5-AZA$\mathrm{dC}$, Sigma-Aldrich) were dissolved in differentiation medium for the first 24 hours and then cultures were switched back to regular differentiation medium and maintained for two weeks. Media was changed every 2 days for the remainder of the experiment. For this study, we performed our analysis on two separate populations of AFS cells.

\subsection{Cardiomyocyte isolation}

Neonatal rat cardiomyocytes (NRCs) were isolated as described previously, with some modifications (Laugwitz et al., 2005). Briefly, hearts from 1-3 day old postnatal pups were 
minced and predigested in $0.7 \mathrm{mg} / \mathrm{ml}$ trypsin-Hank's balanced salt solution (HBSS) without $\mathrm{Ca}^{2+}$ for 16 hours at $4^{\circ} \mathrm{C}$ under mechanical shaking. Following another 4 rounds of 3 minute digestions in collagenase type $2(1 \mathrm{mg} / \mathrm{ml}$ in HBSS $)$ at $37^{\circ} \mathrm{C}$, supernatant which contains single cells was collected, centrifuged at 1000rpm for 10 minutes and then resuspended in fresh medium. After differential plating, in which cells were allowed to attach to tissue culture treated plastic dishes for one hour to separate easily attached fibroblasts, the enriched NRCs were plated on $1 \%$ gelatin coated glass coverslips at a density of $1 \times 10^{4} / \mathrm{cm}^{2}$ and cultured in DMEM/M199 medium (4:1 ratio) with 10\% Horse serum, 5\% FBS and 1\% Penicillin/Streptomycin and 1\% L-glutamine for 48 hours.

\subsection{Co-culture experiment}

hAFS cells were labeled with PKH26 dye (Invitrogen) according to the manufacturer's instructions, and then were co-cultured together by direct contact with NRC at a density of 1 $\times 10^{3} / \mathrm{cm}^{2}$. In all co-culture experiments, the horse serum concentration was reduced to $5 \%$. In indirect co-culture experiment, NRC and hAFS were separated by $3.0 \mu \mathrm{m}$ transwell culture insert (Corning).

\subsection{Dye-transfer experiment}

Two days after seeding, NRCs were labeled using $10 \mu \mathrm{M}$ calcein-AM (Invitrogen) at $37^{\circ} \mathrm{C}$ for 30 minutes. After labeling, cells were washed with PBS three times to remove residual extracellular calcein-AM. PKH26-labeled hAFS cells were added to the culture system. At each time point, the cultures were observed under an epifluorescence microscope (Axio Imager M1 Microscope, Carl Zeiss). In some cases, 50nM 12-O-Tetradecanoylphorbol 13acetate (TPA, Sigma-Aldrich) was added into the culture medium to block dye transfer. As a negative control, NIH $3 \mathrm{~T} 3$ cells were co-cultured with hAFS cells.

\subsection{Immunocytochemistry}

Cells were seeded in chamber slides (NUNC) or on coverslips for immunocytochemistry. Cells were fixed in a $4 \%$ paraformaldehyde solution for 10 minutes and then permeabilized with $0.1 \%$ Triton X-100. Cells were washed three times in PBS and incubated with the following primary antibodies overnight at $4^{\circ} \mathrm{C}$. Rabbit polyclonal anti-SRY-box 2 (Abcam 1:200), rabbit polyclonal anti-cardiac troponin $\mathrm{T}$ (Abcam 1:100), mouse monoclonal antitroponin I (Chemicon 1:100), rabbit polyclonal anti-connexin43 (Invitrogen 1:200) and mouse monoclonal anti-N-cadherin (BD 1:200). After washing, samples were incubated with appropriate fluorescein or rhodamine conjugated secondary antibodies (Jackson Immuno, 1:300) for 45 minutes at room temperature. Nuclei were labeled using either YoPro-3 iodide (Invitrogen) or DAPI (Vector). Samples were mounted and viewed using multitrack high resolution confocal microscopy (Zeiss Axiovert 100M) or epifluoresence microscope (Axio Imager M1 Microscope, Carl Zeiss).

\subsection{Flow cytometry}

For flow cytometry analysis, hAFS cells were cultured as described previously. Cells were washed twice in PBS and incubated with the following fluorochrome-conjugated monoclonal antibodies for 40 minutes on ice: anti-CD34, anti-CD29, anti-ssea4 (R\&D systems), anti-CD44 (eBioscience), anti-CD45, anti-CD90, anti-CD105, anti-CD73, and/or anti-CD146 (Beckton \&Dickinson Pharmingen). After incubation with primary antibodies, cells were washed and the samples were analyzed using a FACSCalibur flow cytometer and CellQuest software (Beckton \&Dickinson). hAFS cells incubated without primary antibodies were used as negative controls, and anti-human HLA-ABC antibody (Beckton $\&$ Dickinson Pharmingen) was used to detect human derived cells. 


\subsection{Reverse transcription polymerase chain reaction (RT-PCR)}

For polymerase chain reaction studies, total RNA was extracted from the cells by Trizol Reagent (Invitrogen). First-strand cDNA was synthesized from RNA by using the SuperScript II reverse transcriptase (Invitrogen) according to manufacturer's protocol. Human cardiac tissue RNA (Ambion) served as a positive control. cDNA samples were subjected to PCR amplification with the following primers: sox2 (Forward:5'-AGT CTC CAA GCG ACG AAA AA-3', Reverse: 5'-GGA AAG TTG GGA TCG AAC AA-3'), cTnT (Forward: 5'-GTG GGA AGA GGC AGA CTG AG-3'; Reverse: 5'-CGA ACT TCT CTG CCT CCA AG-3'), cTnI (Forward: 5'-CCC TGC ACC AGC CCC AAT CAG A-3', Reverse: 5'-CGA AGC CCA GCC CGG TCA ACT-3'), Connexin 43 (5'-GGC GTG AGG AAA GTA CCA AA-3', 5'-CCT CCA GCA GTT GAG TAG GC-3'), N-Cadherin (Forward:5'GCA AGA CTG GAT TTC CTG AAG-3', Reverse: 5'-CTG GAG TTT TCT GGC AAG TTG-3'), H-Cadherin (Forward: 5'-TGC GGA AGA TAT GGC AGA ACT CG-3', Reverse: 5'-GAG TTT TGC CAT TGA CAT CAG TGG-3') Myocyte Enhancer Factor 2C (mef 2C) (Forward: 5'-CTG GGA AAC CCC AAC CTA TT-3', Reverse: 5'-GCT GCC TGG TGG AAT AAG AA-3', Myocyte Enhancer Factor 2D (mef 2D): (Forward: 5'-CAG CAG CCAGCA CTA CAG AG-3', Reverse: 5'-GGC AGG GAT GAC CTT GTT TA-3'). Quantitative PCR were performed using SYBR Green Master Mix Reagents (Applied Biosystems). Expressions were normalized according to expression of GAPDH.

\subsection{Western-Blot analysis}

Cells were washed 3 times with PBS and homogenized in lysis buffer. Protein concentration was determined by DC protein assay (Bio-Rad) using bovine serum albumin as standard. Equal amounts of protein $(100 \mu \mathrm{g})$ were separated by $10 \%$ precast polyacrylamide gel (BioRad) electrophoresis and blotted onto nitrocellulose membranes. After blocking by $1 \%$ Bovine Serum Albumin (BSA) for 1 hour at room temperature (RT), membranes were probed with polyclonal anti sox 2 antibody (Abcam $0.3 \mu \mathrm{g} / \mathrm{ml}$ ) overnight at $4^{\circ} \mathrm{C}$, The filters were washed three times (10 minutes each) at room temperature with blocking solution and then reacted with anti-rabbit secondary antibody conjugated with horse radish peroxidase IgG (cell signaling 1:2000) for 1 hour at RT, washed 3 times and visualized by SuperSignal West Femto Maximum Sensitivity Substrate according to the manufacturer's protocol.

\subsection{Statistical Analysis}

All data where indicated are presented as means \pm SEM, and analyzed using Microsoft Excel 2007.

\section{Results}

\subsection{Stem cell marker expression in hAFS cells}

Expression of stem cell surface markers was assessed in hAFS cells by flow cytometry analysis (Table $1 \&$ supplement data). Consistent with our previous results (De Coppi et al., 2007), hAFS cells expressed a panel of mesenchymal stem cell markers (CD29, CD44, CD73, CD90 and CD 105) but were negative for markers of hematopoietic stem cells (CD45 and CD34). In addition, $94.42 \%$ of the hAFS cells analyzed were positive for CD146 and over $99 \%$ were positive for NG-2, both of which are pericyte markers (Crisan et al., 2008). We also measured the expression of sox2, a marker of embryonic stem/carcinoma cells (Kamachi, Uchikawa, \& Kondoh, 2000) and neural stem cells (Ellis et al., 2004). Immunofluorescence analysis showed that all hAFS cells were positive for sox2 and this result was confirmed by Western Blot (Figure 1A, B). Both cell populations demonstrated similar expression of surface markers. 


\subsection{In vitro differentiation along the cardiac lineage}

In order to determine if hAFS cells could be candidates for differentiation to a cardiac phenotype, we analyzed expression of markers associated with the cardiac lineage. Undifferentiated hAFS cells expressed several cardiac genes, including muscle specific transcriptional factors mef $2 C \& 2 D$, the gap junction protein connexin 43 and the surface anchoring molecules $\mathrm{N}$ - and $\mathrm{H}$-cadherin (Figure 1C). To further induce hAFS cells to differentiate towards a cardiac phenotype, they were cultured in medium containing $10 \mu \mathrm{M}$ 5-AZA-dC for 24 hours, before the 5-AZA-dC was removed and the cells were cultured for additional 10 days in growth media. Phenotypic examination of the cells showed enlargement of the cells and formation of organized stress fibers, consistent with a muscle phenotype. RT-PCR analysis at 5 and 10 days after initiation of differentiation showed a progressive increase in expression of cardiac specific TnI and TnT by 120 and 1200 folds, respectively, and immunostaining confirmed expression of these proteins. In parallel, cardiac differentiation of hAFS cells was accompanied with a reduction in Sox2 expression (Figure 2). Although these results suggest that hAFS cells had differentiated along the cardiac lineage, neither mature sarcomere structure nor spontaneous beating was observed, suggesting an immature cardiac phenotype.

\subsection{Cell-cell contact induced interaction between hAFS cells and rat cardiomyocytes}

We obtained synchronized beating colonies of neonatal rat cardiomyocytes (NRCs) and coculture them with PKH 26 labeled hAFS cells, at a ratio of 1:10 (hAFS:NRCs), for up to 10 days in order to test if hAFS cells can establish functional interactions with the NRCs (Figure 3A). Immunocytochemistry was performed 7 days after initiation of co-culture. The hAFS cells were identified by red PKH26 fluorescence or in some cases by immunostaining with a human specific anti-Nuclear Mitotic Apparatus protein (NUMA) antibody (positive cells demonstrated either purple nuclei or red cytoplasmatic staining). When hAFS were cultured alone, immunostaining with anti-connexin 43 antibodies localized this protein mostly in the cytoplasm, suggesting that it was non-functional (Figure 3B). In contrast, hAFS cells co-cultured with NRCs showed typical membrane-bound connexin 43 staining between adjacent hAFS cells (Figure 3C), as well as between pairs of hAFS/NRC cells (Figure 3D). However, the same transition was not observed when hAFS were co-cultured together with fibroblasts (Figure 3D). Similarly, a re-distribution of N-cadherin, the main component of adherens junctions between cardiomyocytes, from the cytoplasm to the membrane of the hAFS cells was also observed upon co-culture (Figure 3F, G).

\subsection{Functional interaction between hAFS cells and NRCs}

In order to further examine the functionality of gap junction between hAFS cells and NRCS, we performed dye transfer experiments with calcein-AM, a non-fluorescent compound that would transform into a green fluorescent dye after hydrolysis by intracellular esterases. Because of its low molecular weight, the non-permeable calcein could be transferred through intercellular connections such as gap junctions. We co-cultured hAFS cells prelabeled with PKH26 (red) together with NRCs that were loaded with calcein (green). Eighteen hours after initiation of co-culture, several PKH26/calcein double positive cells were observed (Figure 4A, arrows). The green (calcein) fluorescence intensity in PKH26 positive cells was usually weaker than in the fluorescent NRCs, which suggests a passive dye transfer through intercellular connections between hAFS cells and NRCs. For quantification, flow cytometry analysis was carried out. To identify human cells, we used human specific anti HLA-ABC antibody (99.1\% of hAFS cells are positive for HLA-ABC). The number of calcein-positive human cells increased over time, and at 12 hours after initiation of co-culture, over $40 \%$ of human-derived hAFS cells was positive for calcein. This number was significantly reduced in the presence of 12-O-Tetradecanoylphorbol 13- 
acetate (TPA), a gap junction blocker (Figures 4B, C). These results suggest the existence of functional gap junction coupling between hAFS cells and NRCs in co-culture.

\section{Discussion}

Coronary artery disease is the number one cause of death in the world, and is exacerbated by many risk factors including smoking, obesity, hypertension, depression, and family history. Therapies available today can improve the prognosis of patients, but there is currently no treatment modality that will regenerate damaged heart tissue. Cell transplantation has emerged as a potential therapeutic intervention for the treatment of coronary artery disease, and many researchers have focused on development of stem cell therapies for regeneration of cardiac tissue.

Recent studies identified several sub-populations of cardiac cells such as c-Kit ${ }^{+}$(Bearzi et al., 2007), Sca- $1^{+}$cells (Smits et al., 2009) and 'cardiospheres' that directly grow out of cardiac tissue in primary culture (Davis et al., 2009), which display stem cell phenotype. Although cardiac tissue-derived stem cells may represent a natural source of cells to repair damaged heart tissue, their isolation from donor tissue and the difficulty associated with expanding large cell numbers in culture present a significant barrier to clinical use. Other cell sources that hold great potential are human embryonic stem cells and the recently developed iPS cells (Takahashi \& Yamanaka, 2006; Yu et al., 2007). Beating cardiomyocytes can spontaneously derived from these pluripotent cells(Kuzmenkin et al., 2009; Laflamme et al., 2007; Yang et al., 2008). However, teratoma formation have been frequently observed after transplantation of undifferentiated ES (Nussbaum et al., 2007) and enriched ES-derived cardiomyocytes (Hentze et al., 2009). Thus, other stem cells sources that can be easily isolated expanded in culture and hold no safety risks may be a better source for cardiac tissue regeneration. We have recently reported the isolation of stem cells from amniotic fluid (De Coppi et al., 2007). These hAFS cells can be readily obtained are easy to isolate, and require no feeder layers for growth in culture. Unlike other sources of adult mesenchymal stem cells, hAFS cells are highly expansive, and they can differentiate into several lineages. In addition, hAFS cells do not form teratomas upon injection into immune deficient mice.

In the current study, we investigated the applicability of hAFS cells for cellular therapy for cardiac disease. One major goal of this study was to determine whether hAFS cells had the potential to differentiate into cells with a cardiac phenotype. The results show that "naïve" (undifferentiated) hAFS cells express several cardiac-specific genes, and when treated with 5-AZA-dC, the cells respond by increasing the expression of additional cardiac-specific genes. Another goal was to determine if hAFS cells can communicate with authentic cardiomyocytes. Our results show that when hAFS cells are co-cultured with rat cardiomyocytes, a functional communication system is formed between hAFS cells and the rat cardiomyocytes. Specifically, the hAFS cells are able to interact with rat cardiomyocytes through gap junctions, as evidenced by the expression and distribution of gap junction proteins such as connexin 43 in the cells. These data provide significant evidence for the "cardiac potential" of hAFS cells.

In the current study we show, for the first time, expression of sox 2 in hAFS cells. This finding is consistent with the phenotype of porcine AFS cells reported recently (Zheng et al., 2009). Sox 2 plays an important role in the maintenance of pluripotency in embryonic stem cells/embryonic carcinoma cells, and is also an indispensable element for generation of induced pluripotent stem (iPS) cells (Takahashi \& Yamanaka, 2006; Yu et al., 2007). Sox2 expression in hAFS cells was significantly reduced upon their differentiation. hAFS also express a panel of mesenchymal stem cell markers (CD29, CD44, CD73, CD90 and 
CD105). Of particular interest, we found a high level of CD146 (94.42\%) and NG-2 (99.8\%) expression. Both of these proteins are associated with pericytes (Crisan et al., 2008). This suggests that hAFS cells may represent an intermediate state between embryonic and adult mesenchymal stem cells and may point to a possible perivascular origin of the cells. In addition, our study shows that undifferentiated hAFS cells express several cardiac genes, including muscle specific transcriptional factors mef2C and mef2D, together with two surface anchoring molecules N- and H-cadherin (Figure 2). Zhao et al. (Zhao et al., 2005) reported the isolation of a mesenchymal stem cell population from human amnion membrane that express several cardiac markers such as Myosin Light Chain-2a (MLC-2a), Myosin Light Chain-2v (MLC-2v), cTnT, cTnI and the cardiac specific transcription factor GATA4. Others (Chiavegato et al., 2007) showed expression of MLC-2v, $\beta$-Myosin heavy chain, Nkx2.5 and GATA- 4 by these cells. It is possible that hAFS cells and amnion membrane MSC share similar origins; however, there is currently insufficient data to support this hypothesis. These findings, together with the possible perivascular origin of the cells, further support our hypothesis that hAFS cells may be a potential alternative for cardiac cell therapy.

In order to guide the hAFS cells towards a cardiac phenotype we employed an established protocol that uses the chemical compound 5-aza-2'-deoxycytidine (Hakuno et al., 2002). Although the underlying mechanism for its action is still unknown, it is widely used to promote in vitro cardiac differentiation of stem cells, including embryonic (Xu et al., 2002), cardiac (Smits et al., 2009) and mesenchymal stem cells of different origins(Florath et al., 2003; Hakuno et al., 2002). The hAFS cells responded to 24-hour treatment with 5-AZA-dC by reducing their proliferation rate, assuming morphological changes that included cellular elongation, stress fiber reorganization, and up-regulation of the expression of the cardiac genes cTnI and cTnT. However, we recognize that these cells were not functional 'cardiomyocytes' because of the missing of physiological test data. Unlike studies with ES cells and some reports from the differentiation of MSC (Hakuno et al., 2002; Xu et al., 2002), we were not able to detect autonomous beating of differentiated hAFS cells in vitro. One possibility is that the beating phenotype is regulated by a specific group of genes that are not up-regulated in response to 5-AZA-dC treatment in hAFS cells. For example, GATA4 and Tbx5, which have successfully induced ectopic cardiac tissue in mouse embryo, were not detected in hAFS cells before or after 5-AZA-dC treatment (Takeuchi \& Bruneau, 2009). Thus, without spontaneous beating and cardiac specific ionic channel activity, we cannot conclude that hAFS differentiated into mature cardiomyocytes. However, since the cells respond to 5-AZA-dC treatment and acquired partial cardiac phenotype we will continue to search for the necessary elements required to fully differentiate the cells.

Arrhythmogenesis has been a major concern as a side effect of cardiac cell therapy. In clinical trials using skeletal myoblasts for cardiac repair, a higher incidence of ventricular arrhythmia has been documented, although the actual incidence varies (Menasche et al., 2003; Menasche et al., 2008). Although the mechanism underlying the development of arrhythmia after cell therapy has not been fully elaborated, the lack of functional connexin 43 , one of the main ventricular gap junction molecules, and poor coupling with host cardiomyocytes may be involved (Leobon et al., 2003). Therefore, we examined connexin 43 expression in hAFS cells. Our data indicate that undifferentiated hAFS cell express connexin 43 mRNA, but the protein is distributed in a diffuse pattern throughout the cytoplasm, indicating that it is likely non-functional. To assess whether the presence of cardiac muscle could influence the pattern of expression of connexin43, we set up a coculture of hAFS cells and newborn rat cardiomyocytes. Interestingly, we found that a transition to membrane-associated connexin 43 occurred in the hAFS cells adjacent to the cardiomyocytes. On the other hand, hAFS cells cultured with fibroblasts did not show a 
similar transition to membrane-associated connexin43. These results indicate that hAFS cells are able to form connexin 43 junctions with other connexin 43 expressing cells. Similar observations have been found in several other progenitor cells, including endothelial progenitor cells (EPC) (Badorff et al., 2003) and liver stem cells(Muller-Borer et al., 2007), when co-cultured with neonatal cardiomyocytes. Goumans et al. (Goumans et al., 2007) showed similar re-distribution of connexin 43 in adult murine and human cardiac stem cells that were differentiated in vitro. In addition, another recent report showed that skeletal myoblasts modified to express connexin 43 prior to cell injection significantly increased the electrical stability of post-infarction rodent hearts, and this resulted in a protective effect (Roell et al., 2007).

Similarly, the re-distribution of N-cadherin in hAFS cells following co-culture with cardiomyocytes has been observed. We found membrane associated $\mathrm{N}$-cadherin only when hAFS were cultured in the presence of cardiomyocytes. Re-distribution of connexin 43 and $\mathrm{N}$-cadherin could not be observed when hAFS cells were physically separated from the cardiomyocytes in transwell plates that allow soluble cytokines and other signaling molecules to diffuse between the 2 cell types (data not shown), indicating that physical cellcell contacts rather than soluble factors may be responsible for the redistribution of these proteins. N-cadherin is the main component of adherens junctions, and its presence favors the formation and maintenance of gap junctions(Li, Patel, \& Radice, 2006). In addition, Wei et al. (Wei, Francis, Xu, \& Lo, 2005) showed that connexin43 is also part of the N-cadherin multiprotein complex, which is responsible for both the intracellular trafficking of connexin 43 and surface gap junction assembly. Knockdown of N-cadherin by siRNA leads to an almost exclusively cytoplasmic and peri-nuclear distribution of connexin 43 similar to that observed in hAFS cells that were not cultured with cardiomyocytes. The close relationship between connexin 43 and $\mathrm{N}$-cadherin may explain the re-distribution of both proteins in response to the cardiac micro-environment provided in the co-culture experiments.

Additional tests to determine whether the coupling of hAFS cells and cardiomyocytes was functional were carried out. Using dye transfer experiments, we were able to show that fluorescent calcein was transferred from pre-loaded cardiomyocytes to hAFS cells in the coculture experiments. Dye transfer was blocked when the non-specific gap junctional blocker TPA was added. This result supports our conclusion that hAFS cells develop functional intercellular crosstalk with the cardiomyocytes and that this interaction is probably mediated by gap junction proteins such as connexin 43 .

In conclusion, hAFS cells express several cardiac genes prior to differentiation and upregulate other cardiac genes after 5-aza-2'-deoxycitydine induced differentiation. Furthermore, hAFS cells form functional coupling with native cardiomyocytes in an in vitro cardiac micro-environment, which may prevent the development of arrhythmia after treatment. Thus, the hAFS cells, with characteristics shared by embryonic and mesenchymal stem cells and pericytes, may be an appropriate cell source for cardiac cell therapy.

\section{Supplementary Material}

Refer to Web version on PubMed Central for supplementary material.

\section{Acknowledgments}

The authors wish to thank Kenneth Grant in the Wake Forest Microscopy Core Lab for his assistance with imaging, and Dr. Jennifer Olson for editorial assistance with this manuscript. 
Sources of Funding: This research was supported, in part, by a NIH Ruth L. Kirschstein National Research Service Award Individual Fellowship (F31) (\#AA016056-01) (DD), Supported by China Scholarship Council (XG), and Wake Forest Institute for Regenerative Medicine internal department funds.

\section{References}

Badorff C, Brandes RP, Popp R, et al. Transdifferentiation of blood-derived human adult endothelial progenitor cells into functionally active cardiomyocytes. Circulation. 2003; 107:1024-1032. [PubMed: 12600917]

Bearzi C, Rota M, Hosoda T, et al. Human cardiac stem cells. Proc Natl Acad Sci U S A. 2007; 104:14068-14073. [PubMed: 17709737]

Chiavegato A, Bollini S, Pozzobon M, et al. Human amniotic fluid-derived stem cells are rejected after transplantation in the myocardium of normal, ischemic, immuno-suppressed or immuno-deficient rat. J Mol Cell Cardiol. 2007; 42:746-759. [PubMed: 17300799]

Crisan M, Yap S, Casteilla L, et al. A perivascular origin for mesenchymal stem cells in multiple human organs. Cell Stem Cell. 2008; 3:301-313. [PubMed: 18786417]

Davis DR, Zhang Y, Smith RR, et al. Validation of the cardiosphere method to culture cardiac progenitor cells from myocardial tissue. PLoS One. 2009; 4:e7195. [PubMed: 19779618]

De Coppi P, Bartsch G Jr, Siddiqui MM, et al. Isolation of amniotic stem cell lines with potential for therapy. Nat Biotechnol. 2007; 25:100-106. [PubMed: 17206138]

Ellis P, Fagan BM, Magness ST, et al. SOX2, a persistent marker for multipotential neural stem cells derived from embryonic stem cells, the embryo or the adult. Dev Neurosci. 2004; 26:148-165. [PubMed: 15711057]

Florath I, Rosendahl UP, Mortasawi A, et al. Current determinants of operative mortality in 1400 patients requiring aortic valve replacement. Ann Thorac Surg. 2003; 76:75-83. [PubMed: 12842517]

Goumans MJ, de Boer TP, Smits AM, et al. TGF-beta1 induces efficient differentiation of human cardiomyocyte progenitor cells into functional cardiomyocytes in vitro. Stem Cell Res. 2007; 1:138-149. [PubMed: 19383394]

Hakuno D, Fukuda K, Makino S, et al. Bone marrow-derived regenerated cardiomyocytes (CMG Cells) express functional adrenergic and muscarinic receptors. Circulation. 2002; 105:380-386. [PubMed: 11804996]

He Q, Trindade PT, Stumm M, et al. Fate of undifferentiated mouse embryonic stem cells within the rat heart: role of myocardial infarction and immune suppression. J Cell Mol Med. 2009; 13:188201. [PubMed: 18373734]

Hentze H, Soong PL, Wang ST, et al. Teratoma formation by human embryonic stem cells: Evaluation of essential parameters for future safety studies. Stem Cell Res. 2009

Kamachi Y, Uchikawa M, Kondoh H. Pairing SOX off: with partners in the regulation of embryonic development. Trends Genet. 2000; 16:182-187. [PubMed: 10729834]

Kuzmenkin A, Liang H, Xu G, et al. Functional characterization of cardiomyocytes derived from murine induced pluripotent stem cells in vitro. FASEB J. 2009; 23:4168-4180. [PubMed: 19703934]

Laflamme MA, Murry CE. Regenerating the heart. Nat Biotechnol. 2005; 23:845-856. [PubMed: 16003373]

Laflamme MA, Chen KY, Naumova AV, et al. Cardiomyocytes derived from human embryonic stem cells in pro-survival factors enhance function of infarcted rat hearts. Nat Biotechnol. 2007; 25:1015-1024. [PubMed: 17721512]

Laugwitz KL, Moretti A, Lam J, et al. Postnatal is11+ cardioblasts enter fully differentiated cardiomyocyte lineages. Nature. 2005; 433:647-653. [PubMed: 15703750]

Leobon B, Garcin I, Menasche P, et al. Myoblasts transplanted into rat infarcted myocardium are functionally isolated from their host. Proc Natl Acad Sci U S A. 2003; 100:7808-7811. [PubMed: 12805561]

Li J, Patel VV, Radice GL. Dysregulation of cell adhesion proteins and cardiac arrhythmogenesis. Clin Med Res. 2006; 4:42-52. [PubMed: 16595792] 
Menasche P, Hagege AA, Vilquin JT, et al. Autologous skeletal myoblast transplantation for severe postinfarction left ventricular dysfunction. J Am Coll Cardiol. 2003; 41:1078-1083. [PubMed: 12679204]

Menasche P, Alfieri O, Janssens S, et al. The Myoblast Autologous Grafting in Ischemic Cardiomyopathy (MAGIC) trial: first randomized placebo-controlled study of myoblast transplantation. Circulation. 2008; 117:1189-1200. [PubMed: 18285565]

Meyer GP, Wollert KC, Lotz J, et al. Intracoronary bone marrow cell transfer after myocardial infarction: eighteen months' follow-up data from the randomized, controlled BOOST (BOne marrOw transfer to enhance ST-elevation infarct regeneration) trial. Circulation. 2006; 113:12871294. [PubMed: 16520413]

Muller-Borer BJ, Cascio WE, Esch GL, et al. Mechanisms controlling the acquisition of a cardiac phenotype by liver stem cells. Proc Natl Acad Sci U S A. 2007; 104:3877-3882. [PubMed: 17360446]

Nussbaum J, Minami E, Laflamme MA, et al. Transplantation of undifferentiated murine embryonic stem cells in the heart: teratoma formation and immune response. FASEB J. 2007; 21:1345-1357. [PubMed: 17284483]

Roell W, Lewalter T, Sasse P, et al. Engraftment of connexin 43-expressing cells prevents post-infarct arrhythmia. Nature. 2007; 450:819-824. [PubMed: 18064002]

Smits AM, van Vliet $\mathrm{P}$, Metz $\mathrm{CH}$, et al. Human cardiomyocyte progenitor cells differentiate into functional mature cardiomyocytes: an in vitro model for studying human cardiac physiology and pathophysiology. Nat Protoc. 2009; 4:232-243. [PubMed: 19197267]

Takahashi K, Yamanaka S. Induction of pluripotent stem cells from mouse embryonic and adult fibroblast cultures by defined factors. Cell. 2006; 126:663-676. [PubMed: 16904174]

Takeuchi JK, Bruneau BG. Directed transdifferentiation of mouse mesoderm to heart tissue by defined factors. Nature. 2009; 459:708-711. [PubMed: 19396158]

Wei CJ, Francis R, Xu X, et al. Connexin43 associated with an N-cadherin-containing multiprotein complex is required for gap junction formation in NIH3T3 cells. J Biol Chem. 2005; 280:1992519936. [PubMed: 15741167]

Xu C, Police S, Rao N, et al. Characterization and enrichment of cardiomyocytes derived from human embryonic stem cells. Circ Res. 2002; 91:501-508. [PubMed: 12242268]

Yang L, Soonpaa MH, Adler ED, et al. Human cardiovascular progenitor cells develop from a KDR+ embryonic-stem-cell-derived population. Nature. 2008; 453:524-528. [PubMed: 18432194]

Yu J, Vodyanik MA, Smuga-Otto K, et al. Induced pluripotent stem cell lines derived from human somatic cells. Science. 2007; 318:1917-1920. [PubMed: 18029452]

Zhao P, Ise H, Hongo M, et al. Human amniotic mesenchymal cells have some characteristics of cardiomyocytes. Transplantation. 2005; 79:528-535. [PubMed: 15753841]

Zheng YM, Zhao HY, Zhao XE, et al. Development of cloned embryos from porcine neural stem cells and amniotic fluid-derived stem cells transfected with enhanced green fluorescence protein gene. Reproduction. 2009; 137:793-801. [PubMed: 19261834] 
A

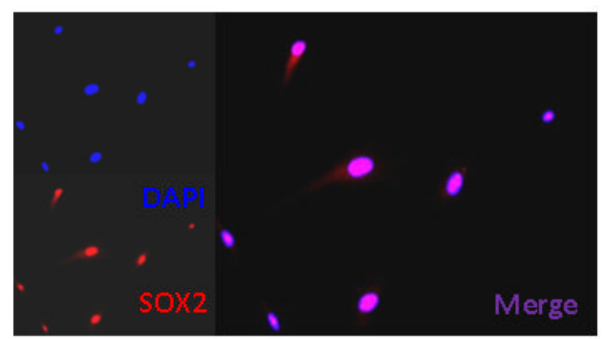

B

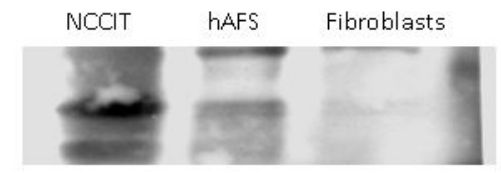

C

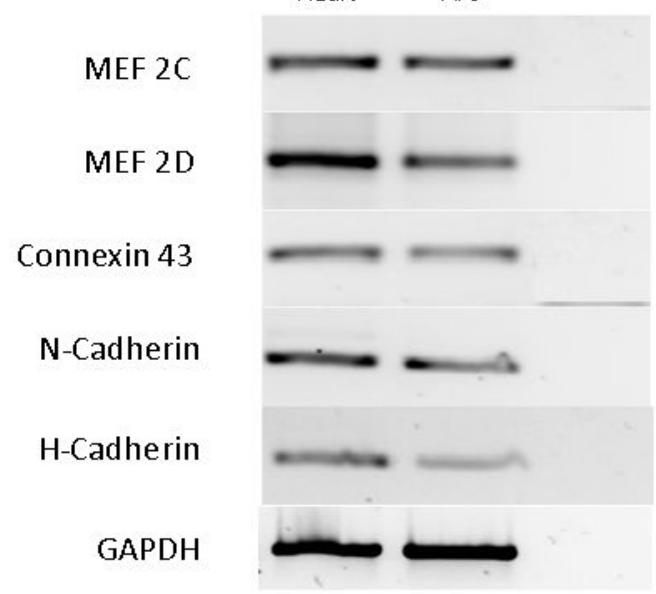

Figure 1. Undifferentiated hAFS cells express the embryonic stem cell marker sox 2 as well as several cardiac genes

(A) Immunocytochemical staining for sox2; (B) Western Blot analysis of sox 2 expression in hAFS cells. Embryonic Carcinoma cell line NCCIT served as a positive control and human fibroblasts served as a negative control; (C) PCR analysis shows that undifferentiated hAFS cells express several cardiac markers including the transcription factors MEF2C and MEF2D, the gap junction protein connexin 43, and the surface proteins $\mathrm{N}$ - and $\mathrm{H}$-cadherin. GAPDH served as an endogenous control. Human heart RNA served as a positive control while RNA without reverse transcription (RT) served as a negative control. 
A

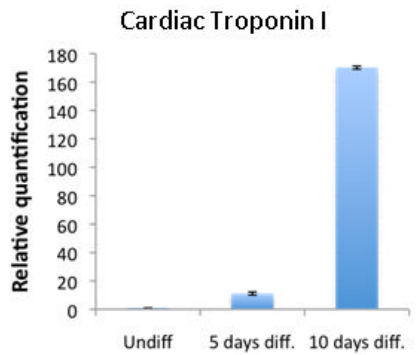

B

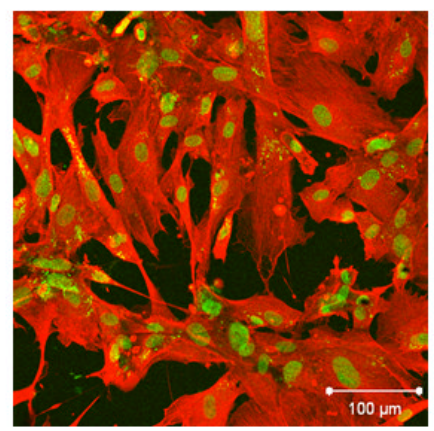

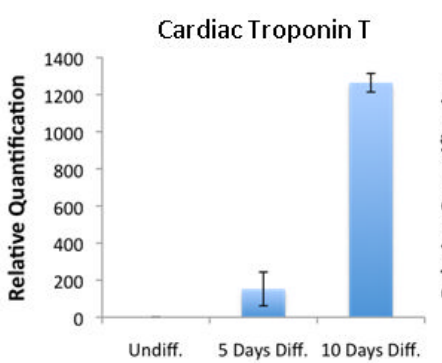
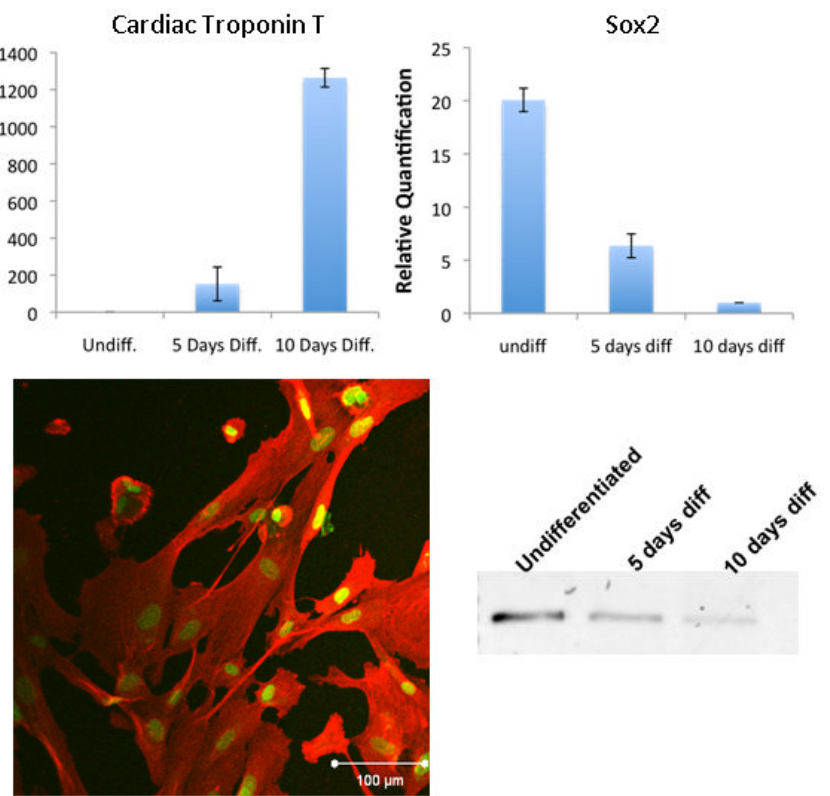

Figure 2. Cardiac differentiation after 5-aza-2'-deoxycitydine treatment

(A) Real-time PCR analysis detects up-regulation of cardiac specific TnT and TnI and down-regulation of the stem cell marker sox2 after 5-aza-2'-deoxycitydine treatment of hAFS cells; (B) Cardiac specific TnT and TnI staining 10 days after initiation of differentiation; (C) End-point PCR analysis shows a gradual decrease of sox2. Values represent means \pm SD AFS cell populations .... 

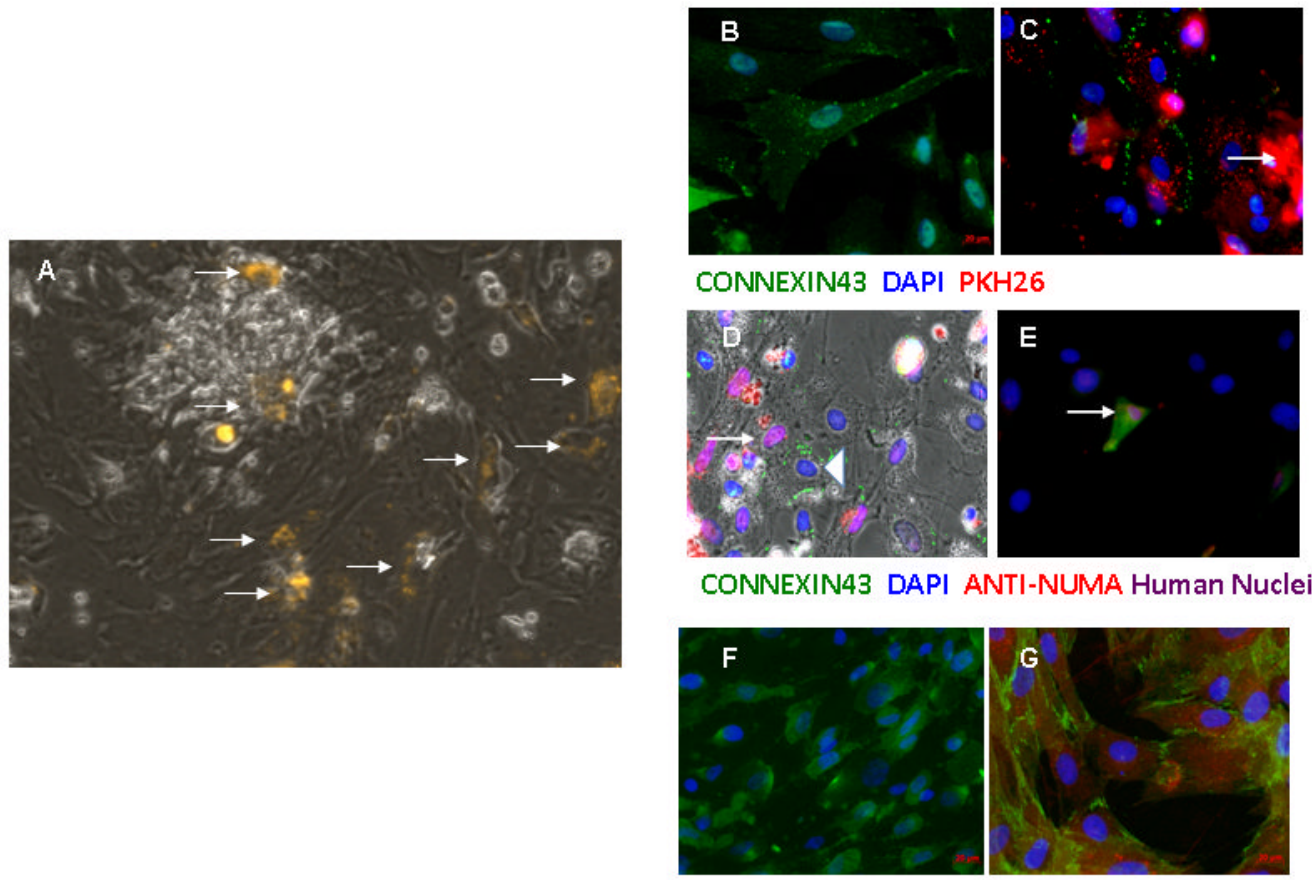

N-CADHERIN DAPI PKH26

Figure 3. Co-culture of hAFS together with hAFS cells

(A) Red fluorescent PKH26 labeled hAFS cells (arrow) were cultured with NRCs. Before co-culture, connexin43 distribution was mainly cytoplasmic and peri-nuclear (B), but this protein became membrane-bound after co-culture. Connexin43 is present between two adjacent PKH26 labeled hAFS cells(C) and between adjacent NRC (triangle) and hAFS cell (arrow). However, when hAFS cells (arrow) were cultured with NIH3T3 fibroblasts (blue nuclei), no re-distribution of connexin43 was observed $(\mathrm{E})$. The same dynamic redistribution process was also found for N-Cadherin when comparing before $(\mathrm{F})$ and after coculture $(\mathrm{G})$. 
A
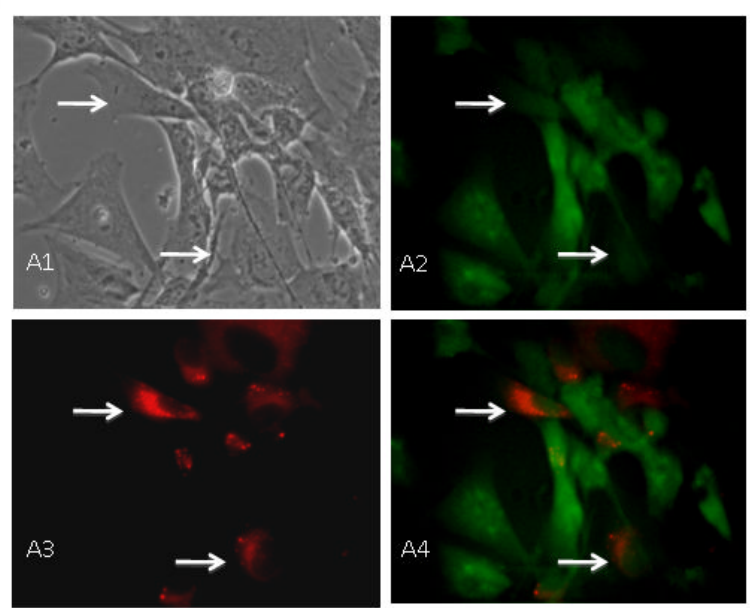
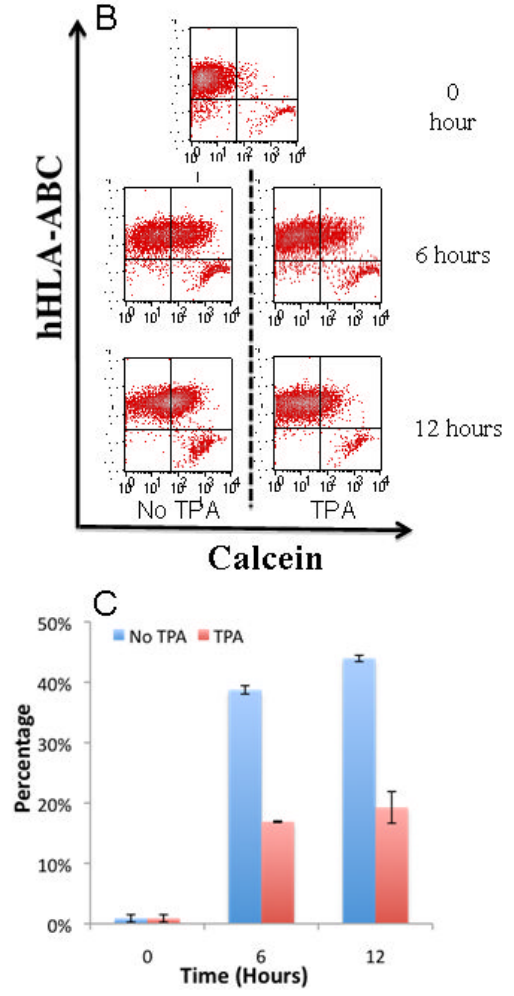

Figure 4. Dye transfer between NRVM and hAFS cells hAFS cells were cultured together with NRVM preloaded with calcein-AM. (A1-A4) Indicate that PKH-26 labeled hAFS cells (arrow) became calcein positive when cultured with NRC. (B) FACS analysis shows that the number of calcein positive human cells (antihHLA-ABC antibody was used to detect human derived cells) increased over time while treatment with TPA partially inhibits the transfer of calcein. (C) Quantitative summary shows calcein positive human cells increased over time while TPA partially blocked the transfer. Values represent means $\pm \mathrm{SD}$ 
Table 1

Surface markers characterization of undifferentiated hAFS cells

Flow cytometry analysis of hAFS cell surface markers.

\begin{tabular}{|c|c|}
\hline CD34 & NA \\
\hline CD45 & NA \\
\hline CD105 & $80.73 \%$ \\
\hline CD73 & $94.92 \%$ \\
\hline CD44 & $98.31 \%$ \\
\hline CD29 & $99.2 \%$ \\
\hline CD90 & $99.4 \%$ \\
\hline SSEA4 & NA \\
\hline HLA-ABC & $99.1 \%$ \\
\hline CD146 & $94.42 \%$ \\
\hline NG-2 & $99.8 \%$ \\
\hline
\end{tabular}

